\section{TITLE: NON-CONTAT ULTRASONIC SPECTROSCOPY MEASUREMENT OF ELASTIC CONSTANTS AND ULTRASONIC ATTENUATION}

\section{SUBMITTED TO: 28th Annual Technical Meeting, Society of Engineering Science, University of Florida, Gainesville, November $6-8,1991$}

R. 8. Schwarz

Tuokkala

W. M. Visscher

\section{DISCLAIMER}

This report was prepared as an account of work sponsored by an agency of the United States Government. Neither the United States Government nor any agency thereof, nor any of their employees. makes any warranty, express or implied, or assumes any legal liability or responsibility for the accuracy. completeness, or usefulness of any information, apparatus, product, or process disclosed, or represents that its use would not infringe privately owned rights. Reference herein to any specific commercial product, process, or service by trade name, trademark, manufacturer. or otherwise does not necessarily constitute or imply its endorsement, recommendation, or favoring by the United States Government or any agency thereof. The views and opinions of authors expressed herein do not necessarily state or reflect those of the United States Government or any agency thereof. 


\title{
NON-CONTACT ULTRASONIC SPECTROSCOPY MEASUREMENT OF ELASTIC CONSTANTS AND ULTRASONIC ATTENUATION
}

\author{
R. B. Schwarz, V.-T. Kuokkala , S. Srinivasan, and W. M. Visscher \\ Center for Materials Science \\ Los Alamos National Laboratory \\ Los Alamos, NM 87545
}

\begin{abstract}
We have developed an ultrasonic spectroscopy method for measuring the elastic constants of solids in hostile environments and over a broad temperature regime. The sample is cut as a rectangular parallelepiped, approximately $1 \mathrm{~mm}^{3}$ in volume. One or two of the sample surfaces are coated with a thin film of a magnetostrictive material such as nickel. The sample is placed coaxially with two solenoids. One solenoid is used to generate an AC magnetic field of small amplitude which stretches the films. By sweeping the frequency of this field, the sample is excited successively into its various mechanical resonance modes. The second solenoid detects the mechanical resonances. The elastic constants are then deduced from the spectrum of mechanical resonances measured at constant temperature. The internal friction is deduced from the width of the resonance peaks.
\end{abstract}

Because the technique is strictly non-contact (the sample may be encapsulated in a fused silica tube), it is ideal for measuring elastic constants in hostile environments or under cortrolled atmospheres. In its present version the system allows us to measure the elastic constants and ultrasonic attenuation of a given sample between 80 and $1000 \mathrm{~K}$. The operation of the system is exemplified by measurements on amorphous $\mathrm{Ni}_{80} \mathrm{P}_{20}$ and crystalline $\mathrm{Ti}_{60} \mathrm{Cr}_{40}$.

- Permanent Address: Institute of Materials Sicience, Tampere University of Technology, Tampere, SF 33101 Finland.

Paper presented at the 28th Annual Technical Meeting, Society of Engineering Science, University of Florida, Gainsville, Florida, November 6-8, 1991. 


\section{INTRODUCTION}

The techniques for measuring the elastic moduli and ultrasonic attenuation of solids may be divided into two broad groups: (a) those based on the measurement of sound known shape. For the first type of measurements measured sound velocity $v$ and the daccurate results require relatively large samples (of the order have various limitations: (a) accurate sometimes unavailable or difficult to prepare; (b) sound of one centimeter), which are sometimes unin piezoelectric transducer to the sample, velocity measurements require attaching a the difference between the thermal expansion coefficients of the sample and from temperature at which the bond was made; heated or cooled to temperatures far aw can be measured for any given sample/transducer and (c) usual

The mechanical resonance method has been extensively applied to thin rods and reeds $[1,2]$. Samples of this shape can be easily excited into longitudinal, flexural, or torsional vibrations, with the mode of vibration determining the type of elastic modulus to be measured. For thin rods of length $L$ and cross section $A$, exciting a thin rod into resonance frequencies are equally spaced. longitudinal vibrations enables the deter $f_{n}=(n /(2 L) \sqrt{ }(E / p)$, where $n$ is an integer. These equally spaced resonance frequencies modulus for each sample and vibration mode.

simple methods give only one elastic modulus fo

Fraser and LeCraw [3], Demarest [4], and Olastic moduli and ultrasonic attenuation ultrasonic spectroscopy method for measuring the between two piezoelectric transducers. Other detects the transmitted ultrasonic signal. The signal of frequency $\omega$, and the other distinct maxima when $\omega$ amplitude of the received signal is usually smeal resonance frequencies. By sweeping the coincides with any of the specimer resonances corresponding to many vibrational modes can be detected in succession. Recently 2 and $350 \mathrm{~K}$. The upper temperature limit of this this system for measurements of plastic components and organic adhesives.

We describe here an ultrasonic spectroscopy technique in which the mathe touching the detection of the mechanical resonances are sample. This technique allows measuremente limit is determined by the availability of approximately $1000 \mathrm{~K}$. The upper temperate the system is ideal magnetostrictive materials of elevated Curie temperatures. In addition, the
for measurements in hostile environments and under controlled atmospheres. 


\section{DESCRIPTION OF THE SYSTEM}

The excitation of mechanical resonances is based on the magneto-mechanical coupling between an alternating magnetic field and a magnetostrictive material. The magnetostrictive material can be the sample itself or, more often, a thin magnetostrictive film deposited onto a non-magnetostrictive sample. To facilitate the calculation of the resonance frequencies (which are then corpared to the measured frequencies), it is convenient to have samples of high symmetry such as cubes, rectangular parallelepipeds, or spheres. In our current applications, the samples are rectangular parallelepipeds with typical dimensions of $1.5 \times 2.0 \times 2.5 \mathrm{~mm}$. For non-magnetostrictive samples, a thin film of a magnetostrictive material (e.g., nickel, cobalt-iron alloy) is deposited onto one or two nonparallel surfaces of the sample.

For measurements, the sample is placed coaxially with two solenoids, $S_{1}$ and $S_{2}$ (Fig. 2). An ac voltage of amplitude $V_{1}$ and frequency $\omega$ is applied to the excitation coil $S_{1}$ to generate a sinusoidal magnetic field $H(\omega t)$ at the sample. Typically, the amplitude of $H$ is 250 A-turn $\mathrm{m}^{-1}$. In addition, a bias magnetic field, $H_{0}$, where $H_{0}>>|H(\omega t)|$, is applied with a permanent magnet or by passing a dc current through the solenoid $S_{1}$. Thus, the magnetic field at the sample is $\mathrm{H}_{\mathrm{O}}+\mathrm{H}(\omega \mathrm{t})$. The bias magnetic field $\mathrm{H}_{0}$ is required to operate the magnetostrictive film in a linear region of stress versus magnetic field.

The magnetostrictive film acts as both a driving transducer and a detecting transducer. As a driving transducer, the film responds to an applied sinusoidal magnetic field, generating a periodic stress, which, for the appropriate value of $\omega$, drives the sample into one of its mechanical resonances. As a detecting transducer, the film responds to the periodic strain in the resonating sample by changing its permeability and thus the inductive coupling between solenoids $S_{1}$ and $S_{2}$. The detailed operation of the system has been described elsewhere [7] and we mention here general principles only.

To decrease the direct coupling between $S_{1}$ and $S_{2}$, detection solenoid $S_{2}$ consists of two identical coils wound clockwise and counterclockwise. (Fig. 2) With the sample removed from the system, the position of $S_{2}$ is adjusted relative to $S_{1}$ so as to minimize the amplitude of $V_{2}$. With the sample inserted in one of the coils of $S_{2}$, there is a slight increase in the inductive coupling between $S_{1}$ and $S_{2}$, which is proportional to the magnetic permeability of the magnetostrictive film. The detection of mechanical oscillations is possible because the permeability of the thin film changes depending on whether the sample is stationary or vibrating.

When the frequency $\omega$ is away from any mechanical resonance, the sample is at rest and the magnetostrictive film is essentially clamped by the sample. Under this condition of zero strain, the film permeability is $\mu^{\epsilon}$. When the frequency $\omega$ coincides with one of the mechanical resonances, and the sample is resonating mechanically (we assume samples of 
low mechanical losses), the thin film has little to do in maintaining the resonances. Then, to a first approximation, the thin film can be considered uncoupled to the sample and vibrating freely. Its permeability is then $\mu^{\sigma}$. Because for magnetostrictive films such as nickel, $\mu^{\epsilon}<\mu^{\sigma}$ the inductive coupling between $S_{1}$ and $S_{2}$ changes when the sample is resonating, changing the amplitude of the signal $\mathrm{V}_{2}$.

The ancillary electronic components for this system are shown in Fig. 2. The function synthesizer providing the sinusoidal driving signal $V_{1}$ to the excitation coil $S_{1}$ is phasemodulated by a triangular signal $h_{1}(\Omega t)$ of frequency $\Omega$ from a second function generator, where $\Omega<<\omega$. Measuring the various mechanical resonance frequencies of the sample requires sweeping the frequency $\omega$ continuously in time. Thus, the driving signal is $V_{1}=V_{10} \cos (\omega t+\varphi)$, where $\omega=\omega_{0}+\alpha t$ and $\varphi=h_{1}(\Omega t)$. In effect, the excitation frequency then alternates with periodicity $\Omega^{-1}$ between two values, $\omega+\Delta \omega$ and $\omega-\Delta \omega$, while increasing linearly with time (Fig. 3).

The signal $V_{2}$ from the detector coil $S_{2}$ is fed to a low-input-capacitance pre-amplifier and is then rectified. The rectified signal has an ac component of frequency $\Omega$, which is detected by a lock-in amplifier working at the same modulation frequency $\Omega$. Thus, the detected signal is the frequency derivative of the amplitude. When the frequency $\omega$ sweeps over one of the sample's mechanical resonances, the detected signal has a clear signature (see Fig. 4 in Ref. [7]). A frequency derivative of a Lorentzian function is fitted to this curve to deduce accurately the resonance frequency and the corresponding attenuation $\mathrm{Q}^{-1}$.

\section{CALCULATION OF THE ELASTIC MODULI}

For the ultrasonic spectroscopy method, the dimensions of the sample are usually of comparable magnitude (Fig. 1). As a result, the resonance frequencies $f_{n}$ are no longer equally spaced. Furthermore, most $f_{n}$ values depend on more than one elastic modulus. The values of $f_{n}$ for a homogeneous solid of known density, dimensions, and elastic moduli can be calculated numerically. There is no analytical solution, however, for the inverse problem of deriving the elastic moduli of this solid from its measured spectrum of resonance frequencies. We thus use an inverse iterative numerical method. The iteration starts with an estimate of the elastic moduli of the sample based on literature data for elastic moduli of similar materials. The $f_{n}$ values are then calculated using the assumed elastic moduli and the known dimensions and density of the sample. The difference between the calculated and measured resonance frequency spectra is quantified by a figure-of-merit function. The global minimum of the function is sought in multidimensional elastic modulus space with computer software developed for the process.[8]

The calculation of the elastic moduli by the iterative process described above requires that we match each of the measured resonances with one of the calculated resonances. This 
may be a difficult task because: (a) an erroneous assumption for the initial values of the sample's elastic moduli may result in the permutation of a pair of calculated resonances with respect to the measured ones, and (b) one or more of the mechanical resonances are sometimes not detected. Missing one or more of the mechanical resonances in the measured spectrum is of no great consequence because the number of measured resonances usually largely exceeds the number of elastic constants to be determined. A method for identifying the resonance modes was recently developed by Stekel et al . [9]

\section{MEASUREMENTS}

We are using the ultrasonic spectroscopy system to measure the second order elastic moduli and the ultrasonic attenuation of various metallic alloys. As examples, we present measurements for works in progress.

\section{(a) Measurements in amorphous $\mathrm{Ni}_{80} \mathrm{P}_{20}$}

Rectangular parallelepiped shaped samples, $3.17 \times 2.37 \times 1.83 \mathrm{~mm}$, were cut from a electrodeposited rod of amorphous $\mathrm{Ni}_{80} \mathrm{P}_{20}$ using a thin diamond blade. Following a light mechanical polishing, thin nickel films (approximately $5 \mu \mathrm{m}$ thick) were electrolytically deposited onto three non-parallel faces to enable the excitation and detection of the mechanical resonances.

Figure 4 shows the temperature dependence of the bulk and shear moduli of amorphous $\mathrm{Ni}_{80} \mathrm{P}_{20}$ normalized to the value of these moduli at $79 \mathrm{~K}$. These measurements were taken after annealing the sample at $520 \mathrm{~K}$ for 275 minutes followed by a slow cooling. This anneal relaxes the alloy and for all subsequent measurements done at $T<520 \mathrm{~K}$, the amorphous alloy is in an isoconfigurational state. At each test temperature we measured between 12 and 15 mechanical resonances, a number that largely exceeds that necessary to determine the two independent elastic moduli of the amorphous alloy. The elastic moduli in Fig. 4 have not been compensated for thermal expansion. Because the coefficient of thermal expansion for $\mathrm{Ni}_{80} \mathrm{P}_{20}$ is $12 \times 10^{-6}$, [10] we estimate that the thermal expansion compensation would change the elastic moduli by less than $0.24 \%$. Except for the single measurement at $520 \mathrm{~K}, \mathrm{~B}$ has a linear temperature dependence. In contrast to this, - dG/dT increases with increasing temperature.

Because measurements in the present magnetostrictive system the are done without touching the sample, the resonant frequencies and ultrasonic attenuation are quite reproducible. This enables us to remove the sample from the ultrasonic system (to perform other measurements which do not perturb the state of the sample, such as X-ray diffraction) and continue the measurements at a later time. Figure 5 illustrates this capability. The figure shows the resonance frequency for one of the mechanical resonance modes of amorphous $\mathrm{Ni}_{80} \mathrm{P}_{20}$ as a function of time during an isothermal anneal in argon at $521 \mathrm{~K}$. At points $A, B$, and $r$, the sample was removed from the system to study its structure by $x$ - 
ray diffraction. The thin lines traced through the data help visualize two processes. For annealing time $<1000$ minute, the resonance frequency has a slow monotonic increase. The X-ray diffraction patterns taken at points $A$ and $B$ showed that the sample was amorphous. After an annealing time close to 1000 minutes, the frequency starts increasing at a much faster rate. An $x$-ray diffraction pattern taken at $\mathbf{C}$ shows Bragg peaks indicating that the change in slope corresponds to the onset of crystallization. The resonance frequency continues to increase during crystallization and approaches a saturation for $\log (t)>4$. All other resonance frequencies showed a similar time dependence. In this material, crystallization is most likely homogeneous and the observed time delay for the initiation of crystallization is the time necessary to establish an equilibrium population of crystallization embryos (transient nucleation, [11]). The cyrstallization process also gives clear signatures in the ultrasonic attenuation. These measurements will be discussed separately.

\section{(b) Measurements in $\mathrm{Ti}_{60} \mathrm{Cr}_{20}$}

In 1953, Blatter and von Allmen [12] reported a massive reversible cyrstal-to-amorphous transformation near $770 \mathrm{~K}$ between a (metastable) bcc-Ti60 $\mathrm{Cr}_{40}$ alloy and and amorphous alloy of the same composition. Although this solid-state amorphization reaction has not been reproduced at other laboratories, TEM and differential scanning calorimetry studies suggest that unexpected crystal-to-crystal transformations do take place near this temperature. These transformations are being studied theoretically [13] and experimentally $[14,15,16]$. Figure 7 shows the ultrasonic attenuation in polycrystalline bcc-Ti $60 \mathrm{Cr}_{40}$. A cm-size alloy ingot was prepared by melting the elements in a watercooled silver boat by induction heating. The cooling rate following the removal of the RF power is sufficiently fast to solidify the alloy in the bcc solid solution phase which is metastable below $1200^{\circ} \mathrm{C}$. [17] Samples for the ultrasonic measurements were cut from this ingot and were coated with a thin film of Fe50 Co50. The data in Fig. 6 was obtained while increasing the temperature. The solid curve is a spline fit to the data. There are two attenuation peaks, with onsets at $220 \mathrm{~K}$ and $840 \mathrm{~K}$, identified as peaks $A$ and B. Peak $A$ is reversible; we do not have yet an interpretation for this peak. Peak B is irreversible. An $X$-ray diffraction pattern of the sample, taken at room temperature after heating the sample past this peak, revealed that the sample had crystallized into a two-phase mixture of hcp-Ti and $\alpha-\mathrm{TiCr}_{2}$ (C15-structure Laves phase). The equilibrium phase diagram of $\mathrm{Ti}-\mathrm{Cr}$ [17] gives the eutectoid temperature for the reaction $\alpha-\mathrm{Ti}+\alpha-\mathrm{TiCr}_{2} \rightarrow \beta-\mathrm{Ti}+\alpha-\mathrm{TiCr}_{2}$ at $940 \mathrm{~K}$. The present data shows that on heating the metastable single phase $\beta-\mathrm{Ti}$, it starts transform at $840 \mathrm{~K}$, possibly into a mixture of $\alpha-\mathrm{Ti}+\alpha-\mathrm{TiCr}_{2}$. Because the data was obtained at a varying heating rate (determined by the time necessary to take the measurements at each test tmeperature), this peak can have arbitrary shape. The fast increase in the attenuation for $T>980 \mathrm{~K}$ is caused by onset of the magnetic transformation of the Fe-Co thin film, which ocurrs near $1000 \mathrm{~K}$. [17] 
This work was supported by the US Department of Energy, Office of Basic Energy Sciences. The work of V.-T. Kuokkala was partially supported by the Tampere University of Technology. We thank A. Mayer for preparing amorphous $\mathrm{Ni}_{80} \mathrm{P}_{20}$ by electrodeposition, and $\mathrm{P}$. Yvon for the preparation of the TiCr alloy.

\section{REFERENCES}

1. J. Marx, Rev. Sci. Instrum. 22, 503 (1951).

2. R. B. Schwarz, Rev. Sci. Instrum. 48, 111 (1977).

3. D. B. Fraser and R. C. LeCraw, Rev. Sci. Instrum. 35, 1113 (1964).

4. H. Demarest, J. Acoust. Am. 49, 768 (1971).

5. I. Ohno, J. Phys. Earth 24, 355 (1976).

6. A. Migliori, W. M. Visscher, S. Wong, S. E. Brown, I. Tanaka, H. Kojima, and P. B. Allen, Phys. Rev. Lett. 64, 2458 (1990).

7. V. -T. Kuokkala and R. B. Schwarz, Submitted to Rev. Sci. Instrum. (1991).

8. W. M. Visscher, J. Accoust. Soc. Amer. 90, 2154 (1991).

9. A. Stekel, J. L. Sarrao, T. M. Bell, R. G. Leisure, W. M. Visscher, and A. Migliori, submitted to $\mathbf{x x x}$

10. G. Dietz and F. -J. Klein, J. Non-Cyyst. Solids 89, 290 (1967).

11. M. G. Scott, in Amorphous Metallic Alloys, edited by F. E. Luborsky (Butterworths, London, 1983), ch. 10.

12. A. Blatter and M. von Allmen, Phys. Rev. Letters 54, 2103 (1985).

13. M. Sluitter and P. E. A. Turchi, Mater. Res. Soc. Symp. Proc., 186, 95 (1991). Phys. Rev. B43, 12251 (1991).

14. R. Prasad, R. F. Somekh, and A. L. Greer, Mat. Sci and Engng. A133, 6060 (1991).

15. W. Sinkler and D. E. Luzzi, $\mathbf{x x x}$

16. A. Blatter, N. Koch, and U. Kambli, J. Less-Common Metals 145, 81 (1988).

17. Binary Alloy Phase Diagrams, edited by T. B. Massalski, J. L. Murray, L. H. Bennett, and H. Baker (Amer. Soc. for Metals, Metals Park, OH, 1986), Vol.1 p. 873. 


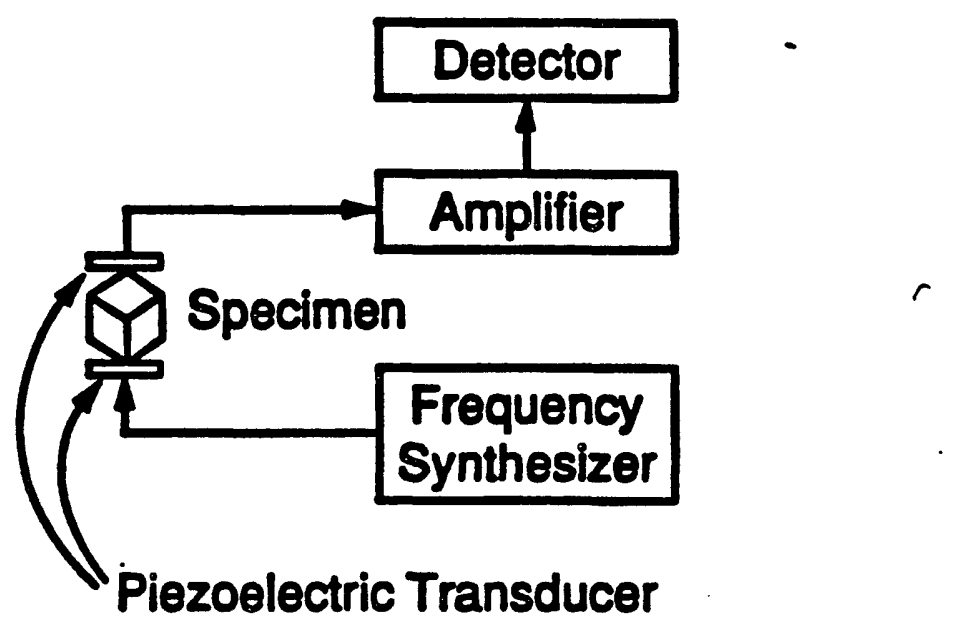

Fig. 1. Schematic of the two-piezoelectric transducer system for measuring the mechanical resonance spectrum of a small sample (after Ref. 5). 


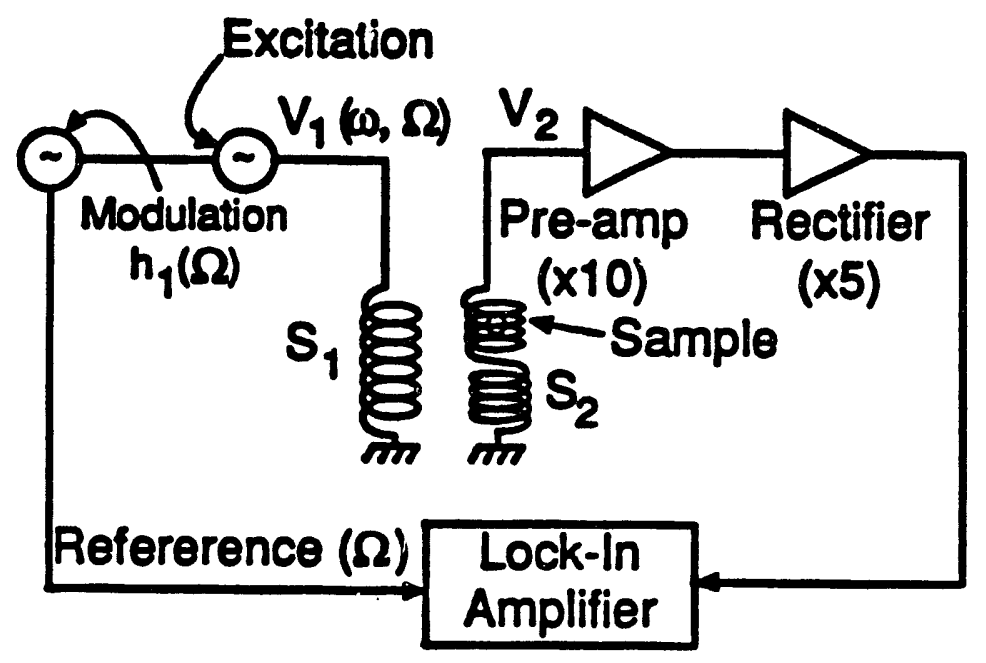

Fig. 2. Schematic of the magnetostrictive excitation/detection system for measuring the mechanical resonance spectrum of a small sample. Solenoids $S_{1}$ and $S_{2}$ are concentric with each other and with the sample. 


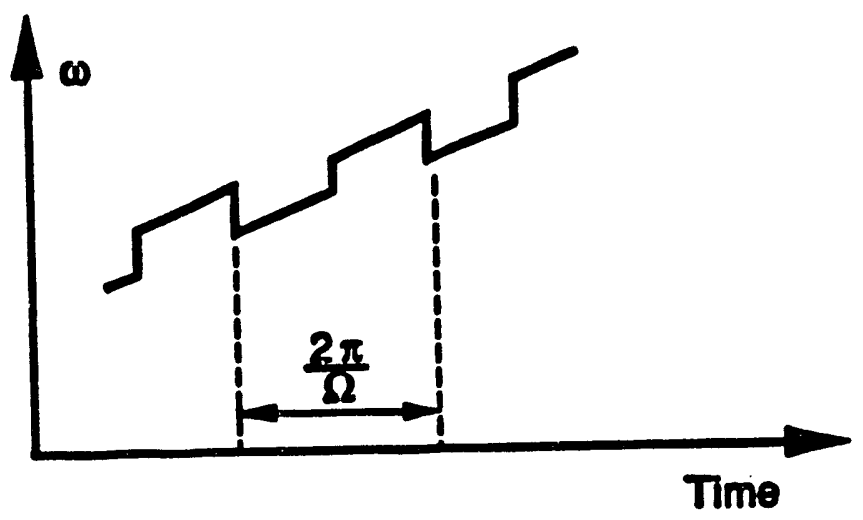

Fig. 3. Time dependence of the excitation frequency in the system shown in Fig. 4. 


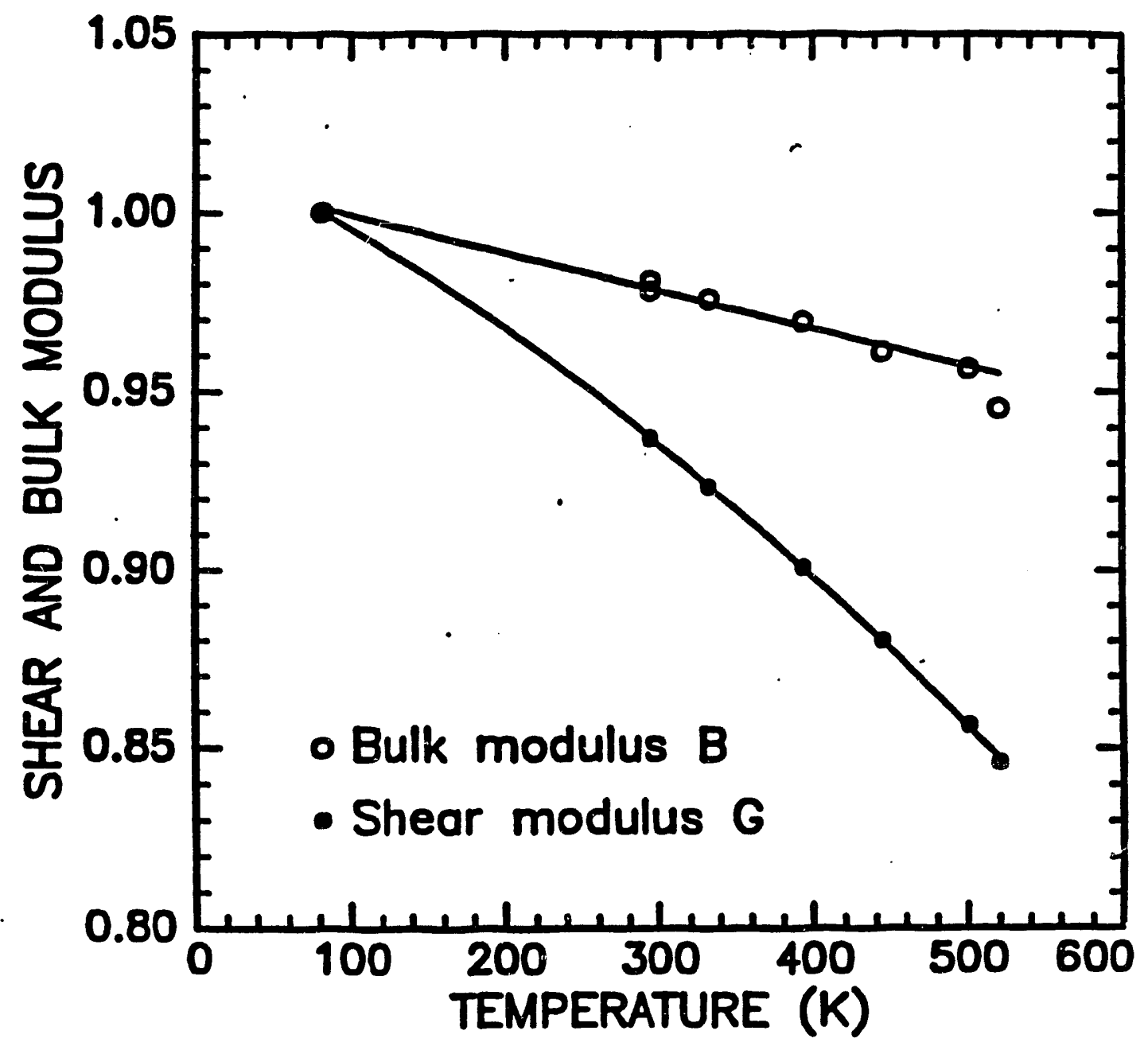

Fig. 4. Bulk modulus $B$ and shear modulus $\mathbf{G}$ of amorphous $\mathrm{Ni}_{80} \mathbf{P}_{20}$, measured as a function of temperature. The measurenents have been normalized by the values at $80 \mathrm{~K}, \mathrm{~B}(80)=170.6 \mathrm{GPa}$ and $\mathrm{G}(80)=44.4 \mathrm{GPa}$. 


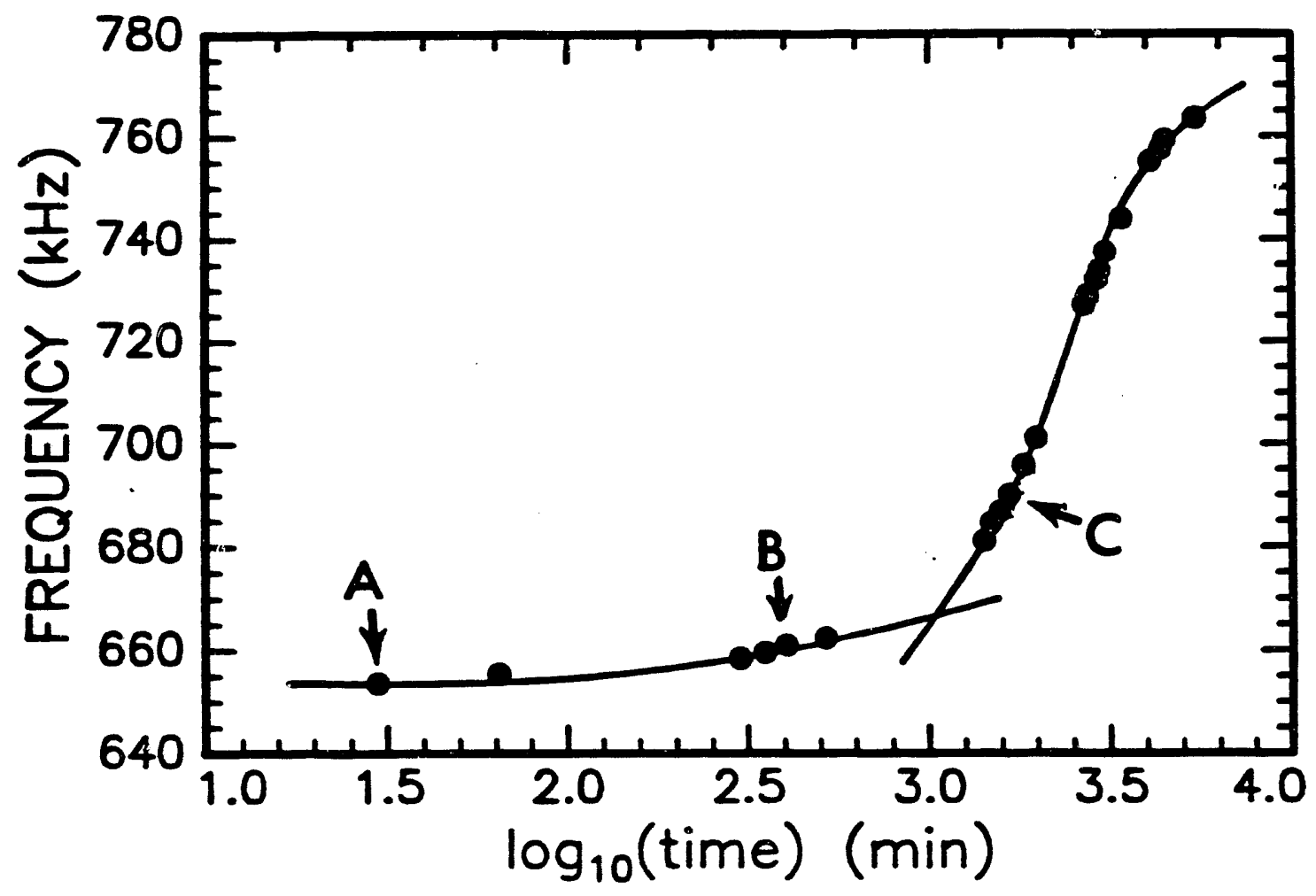

Fig. 5. Resonance frequency for one of the normal modes of amorphous $\mathrm{Ni}_{80} \mathrm{P}_{20}$ as a function of annealing time at $521 \mathrm{~K}$. 


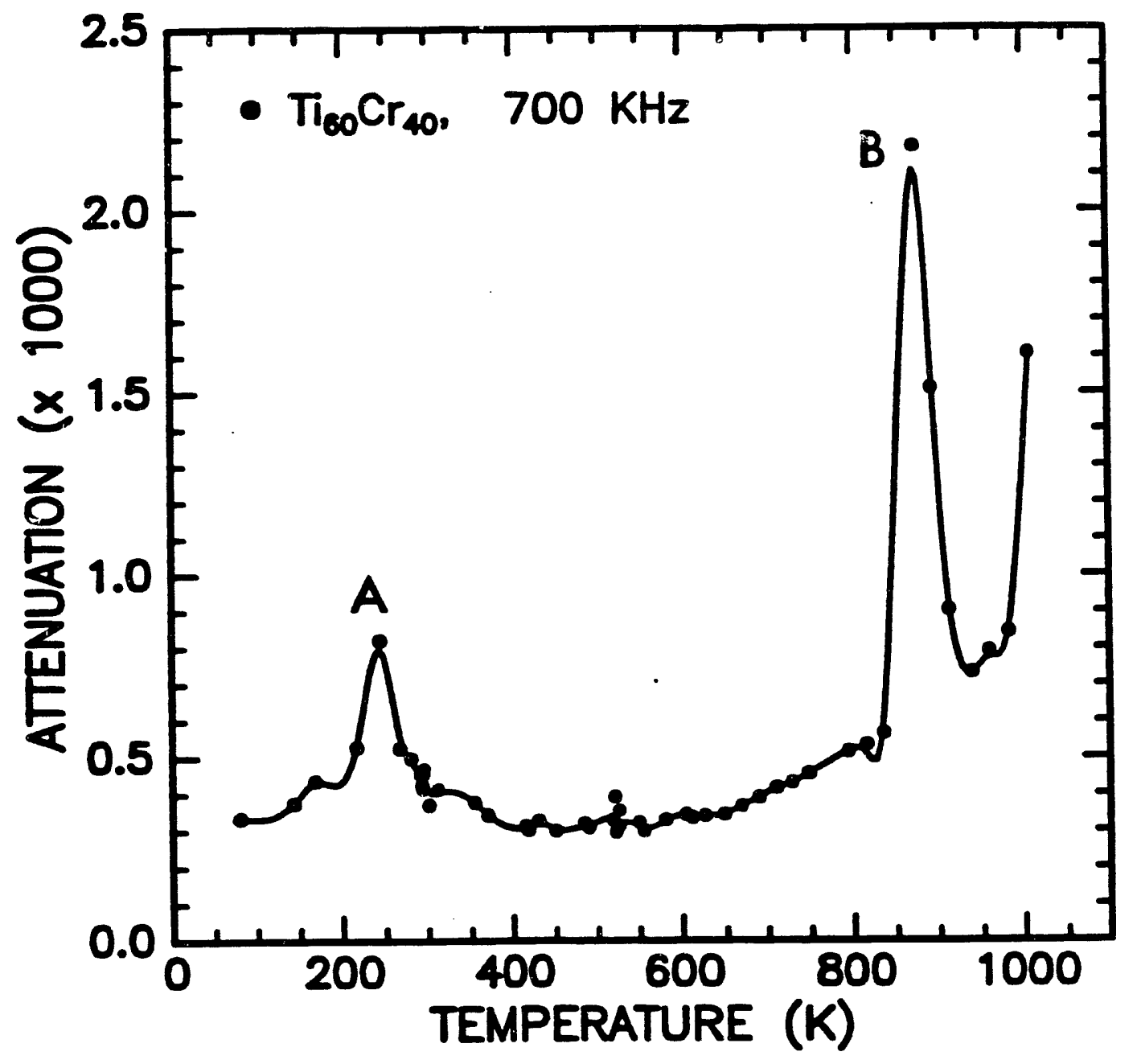

Fig. 6. Ultrasonic attenuation of metastable polycrystalline boc-Ti60 Ti40 measured following stepwise increases in temperature. Sample resonating in a normal mode close to $700 \mathrm{kHz}$. 

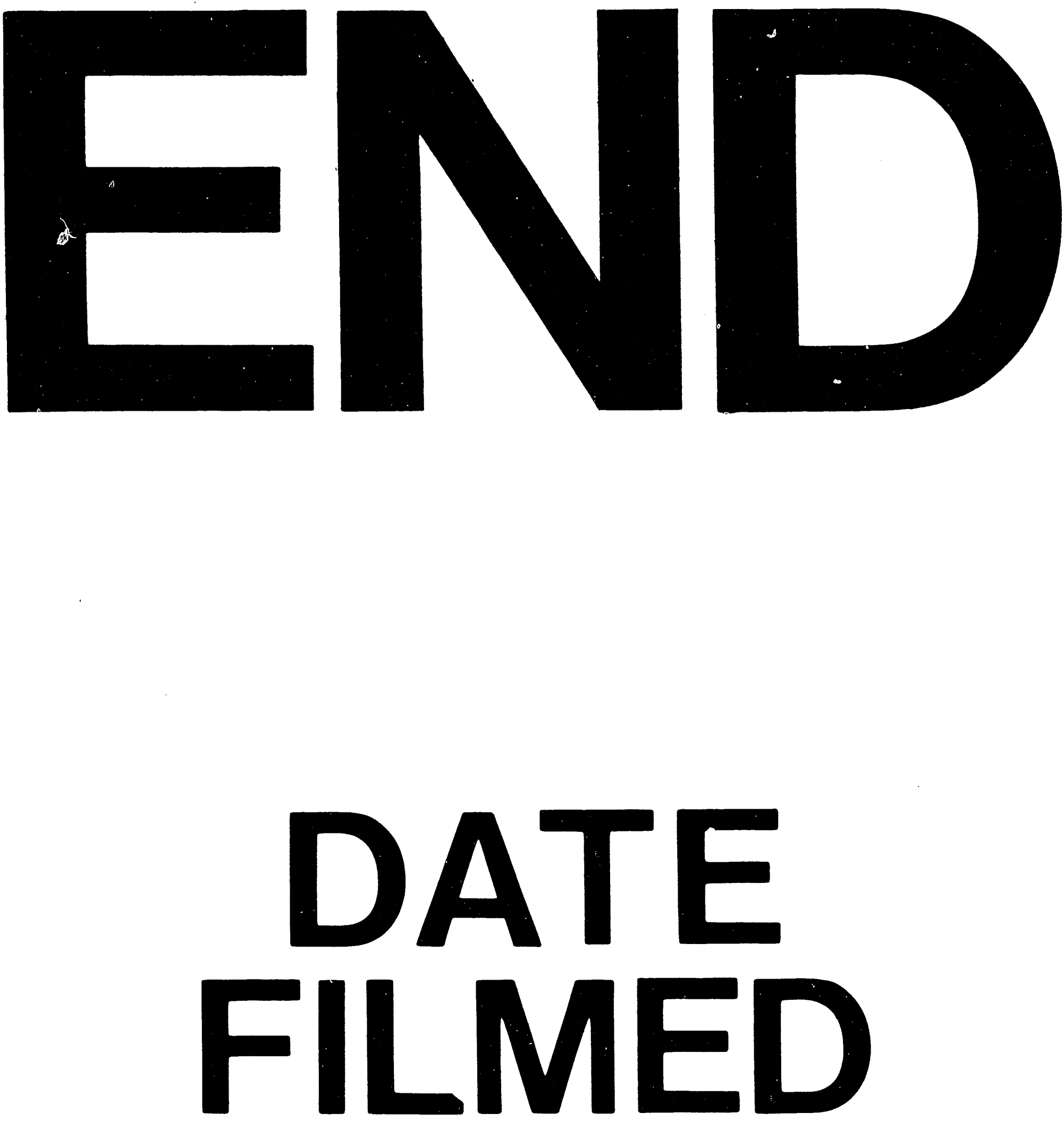

1

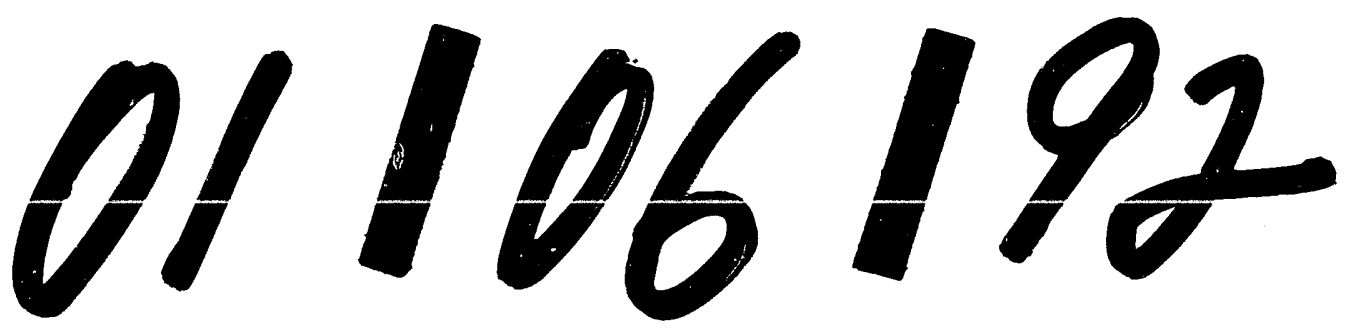


